\title{
谵 \\ Perspectives for heavy flavour production in ATLAS
}

\author{
Evelina Bouhova-Thacker
}

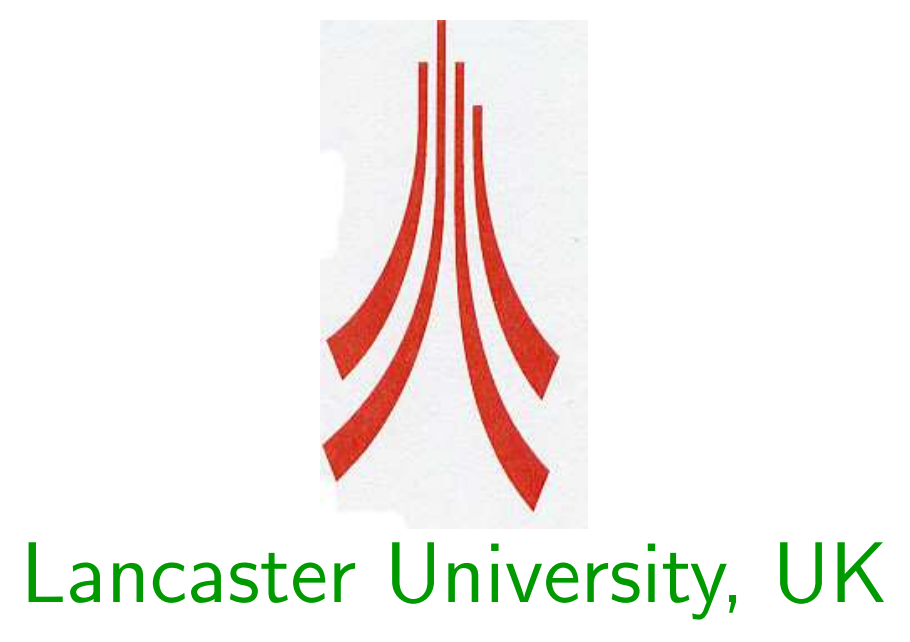

DIS 2008, 7-11 April 2008, University College London 


\section{The ATLAS Detector}

Multipurpose experiment designed to search for new particles at mass scales $\mathcal{O}(1 \mathrm{TeV})$ and new phenomena based on high- $p_{T}$ objects

$\downarrow$ Lepton reconstruction and identification over a wide range of $p_{T}$

- Electrons: from $p_{T}>0.5 \mathrm{GeV}$ in the outer part of the Inner Detector (ID) - the Transition Radiation Tracker, for $p_{T}>4 \mathrm{GeV}$ additional identification from the Electromagnetic Calorimeter (EM)

- Muons: for $p_{T}>6(4) \mathrm{GeV}$ combined information from the Muon Spectrometer (MS) and the Inner Detector

$\checkmark$ Jet reconstruction in the Electromagnetic and Hadronic Calorimeters

$\checkmark$ Charged tracks $\left(p_{T}>0.5 \mathrm{GeV},|\eta|<2.5\right)$ and vertex information provided by the Inner Detector

Design luminosity $\mathcal{L}=10^{34} \mathrm{~cm}^{-2} \mathrm{~s}^{-1}$, early data at $\mathcal{L}=10^{31} \mathrm{~cm}^{-2} \mathrm{~s}^{-1}$

Heavy flavour studies presented here were done with early data and/or at low luminosity $\mathcal{L}=10^{33} \mathrm{~cm}^{-2} \mathrm{~s}^{-1}$ 
\ At $14 \mathrm{TeV} \sigma_{b \bar{b}} \sim 500 \mu b, 40 \mathrm{MHz}$ rate, $1 b \bar{b}$ in $100 p p$ collisions

$\checkmark$ With $10 \mathrm{pb}^{-1}$, i.e. one month of running expect about $2 \times 10^{8}$ beauty and $3 \times 10^{5} \mathrm{~J} / \psi$ events selected by the first level trigger (LVL1)

$\downarrow 10-100 \mathrm{pb}^{-1}$ B-Physics and quarkonia signatures used to

- understand detector properties and trigger system

- measure total and differential production cross-sections $\left(\sigma_{b \bar{b}}, \sigma_{J / \psi}, \sigma_{\Upsilon}\right.$ and $B^{+} \rightarrow J / \psi K^{+}$), polarisation of $J / \psi$ and $\Upsilon$, observe $\chi_{c}$ and $\chi_{b}$ decays, inclusive and exclusive B-hadron decay measurements

\ $200 \mathrm{pb}^{-1}-1 \mathrm{fb}^{-1}$ ( currently at the Tevatron) - improve measurements of B-hadron properties, set new limits for discovery channels

$\downarrow 10-30 \mathrm{fb}^{-1}$ full B-physics potential reached 


\section{Trigger Strategies}

LVL1: $40 \mathrm{kHz}, 2.5 \mu \mathrm{s}$

$\rightarrow$ Muon triggers

- single-muon trigger: $\mathcal{L}=10^{33} \mathrm{~cm}^{-2} \mathrm{~s}^{-1} \quad p_{T}>6$ - $8 \mathrm{GeV}(1 \mathrm{MU} 6,1 \mathrm{MU} 8)$ $\mathcal{L}=10^{31} \mathrm{~cm}^{-2} \mathrm{~s}^{-1} \quad p_{T}>4 \mathrm{GeV}(1 \mathrm{MU} 4)$

- di-muon trigger: $p_{T}^{\mu_{1}}>6 \mathrm{GeV}, p_{T}^{\mu_{2}}>4 \mathrm{GeV}(2 \mathrm{MU} 6)$

$\downarrow J / \psi / \Upsilon \rightarrow e^{+} e^{-}$triggered by $2 \mathrm{EM}$ clusters with $E_{T}>3$ (5) GeV, 2EM3 (2EM5) at $\mathcal{L}=10^{31} \mathrm{~cm}^{-2} \mathrm{~s}^{-1}$ and by $1 \mathrm{MU} 6$ at $\mathcal{L}=10^{31}-10^{33} \mathrm{~cm}^{-2} \mathrm{~s}^{-1}$

LVL2: $1-2 \mathrm{kHz}, 40 \mathrm{~ms}$ - confirm and refine LVL1 objects, search for additional signatures in LVL1 Regions of Interest (Rol)

Single-muon: search in wider LVL1 Rol

$\checkmark$ Di-muon: search in two LVL1 Rol

cross sections usually orders of magnitude smaller than for single-muons of the same $p_{T}$ Event Filter (EF): $200 \mathrm{~Hz}, 4 \mathrm{~s}$ - uses complete event data, "offline-like" algorithms to define complex signatures for final selection 


\section{Inclusive $b$ Cross Section Strategy I}

Based on "Quarkonium trigger": $b \bar{b} \rightarrow J / \psi\left(\mu^{+} \mu^{-}\right) X \quad \mathcal{L}=10^{33} \mathrm{~cm}^{-2} \mathrm{~s}^{-1}$ invariant mass within a $3 \sigma$ window

$\sigma_{J / \psi}=56 \mathrm{MeV}, \sigma_{\Upsilon}=180 \mathrm{MeV}$

Background rates: $J / \psi 6 \mathrm{~Hz}, \Upsilon 13 \mathrm{~Hz}$

Signal rates:

LVL1 1 MU6 $J / \psi 20 \mathrm{~Hz}, \Upsilon 3 \mathrm{~Hz}$

LVL1 2MU6 $J / \psi 7 \mathrm{~Hz}, \Upsilon 1 \mathrm{~Hz}$

Fraction of $J / \psi$ from B-decays: $33.7 \%$

Offline selection: detached $J / \psi$ vertex

$\checkmark \Delta R$ cut - combinatorial background

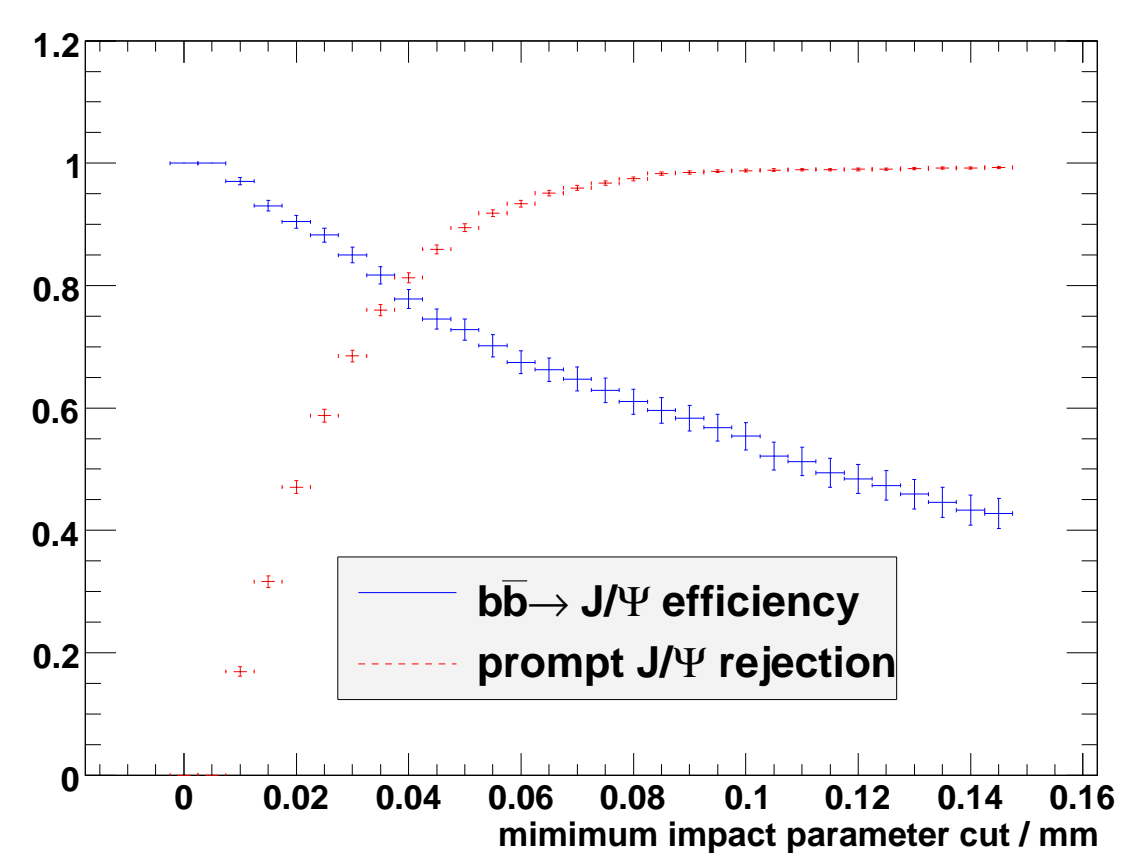

$\checkmark$ Impact parameter cut - prompt background

$\epsilon_{J / \psi} \sim 60 \% \quad$ background rejection $r_{\text {prompt }} \sim 100 \%$

$$
\begin{array}{lll}
N_{J / \psi}^{\text {sel,sig }}=N_{J / \psi}^{\text {trig }} \times 0.1005 & \mathrm{~S} / \mathrm{B} \approx 1.53 & (J / \psi) \\
N_{\Upsilon}^{\text {sel,sig }}=N_{\Upsilon}^{\text {trig }} \times 0.4329 & \mathrm{~S} / \mathrm{B} \approx 0.33(\Upsilon)
\end{array}
$$




\section{Inclusive $b$ Cross Section Strategy II}

\section{Based on "b-Jet trigger"}

LVL1: $1 \mathrm{MU} 6+1 \mathrm{~J} 17$ (jet with $E_{T}>17 \mathrm{GeV}$ )

LVL2: $E_{T}^{\text {jet }}>30 \mathrm{GeV}$, cut on b-jet weight

$\epsilon^{\text {trig }}=13.5 \%$, b-purity $p_{b}=23 \%$

with b-jet weight cut

$\epsilon^{\text {trig }}=7.5 \%$, b-purity $p_{b}=36 \%$

Offline selection: jet selection based on

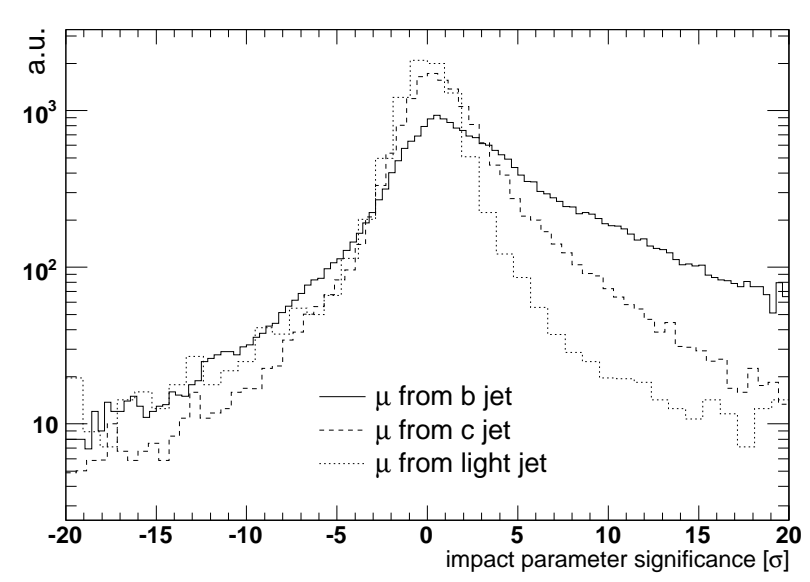

$\checkmark$ life-time tag (impact parameter)

$\downarrow \mu$-tag $\left(p_{T}^{r e l}: p_{T}^{\mu}\right.$ relative to jet axis)

$\epsilon^{r e c}=85 \%$

Fractions of $b$-jets and background jets determined by fitting the simulated $p_{T}^{r e l}$ distributions to the data sample $b$-fraction: $(23 \pm 2) \% \quad$ background: $(77 \pm 4) \%$

$$
N_{b \rightarrow \mu}^{\text {sel }}=N_{\mu-(b) j e t} \times 0.0264(0.0147)
$$

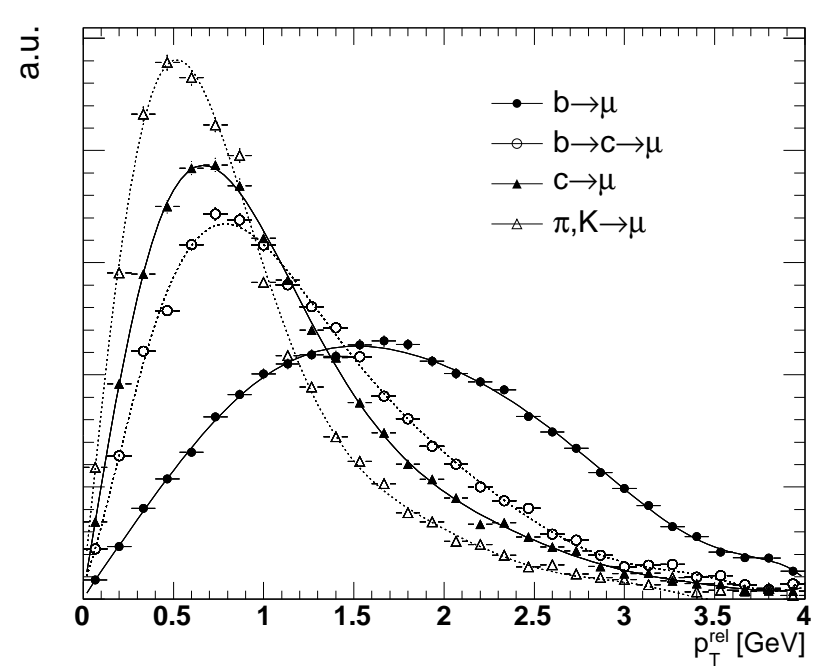




\section{b-Production Cross Section}

$\checkmark$ Expected number of signal events $\left(\mathcal{L}=10^{33} \mathrm{~cm}^{-2} \mathrm{~s}^{-1}\right)$

\begin{tabular}{|l|r|r|r|r|}
\hline & prescale & $0.01 \mathrm{fb}^{-1}$ & $0.1 \mathrm{fb}^{-1}$ & $1 \mathrm{fb}^{-1}$ \\
\hline$b b \rightarrow J / \psi(\mu 6 \mu 4)$ with $1 \mu$ LVL1 & 10 & 2200 & 22000 & 220100 \\
\hline$b b \rightarrow J / \psi(\mu 6 \mu 4) X$ with $2 \mu$ LVL1 & 10 & 700 & 7300 & 72900 \\
\hline$b b \rightarrow \Upsilon(\mu 6 \mu 4) X$ with $2 \mu$ LVL1 & 10 & 400 & 4300 & 43300 \\
\hline$b b \rightarrow \mu(6)+b$-jet & 1000 & 900 & 9000 & 90300 \\
\hline
\end{tabular}

$\checkmark$ Estimated time for $\mathcal{O}(1 \%)$ measurement (statistical error only)

\begin{tabular}{|l|c|c|c|}
\hline & \multicolumn{3}{|c|}{ specific luminosity $\left[\mathrm{cm}^{-2} \mathrm{~s}^{-1}\right]$} \\
channel & $\mathcal{L}=10^{31}$ & $\mathcal{L}=10^{32}$ & $\mathcal{L}=10^{33}$ \\
\hline$b b \rightarrow J / \psi(\mu 6 \mu 4) X$ & 1 year (PS 1) & 1 month (PS 1) & 1 month (PS 10) \\
$b \bar{b} \rightarrow \mu(6)+b$-jet & 1 month (PS 10) & 1 month (PS 100) & 1 month (PS 1000) \\
\hline
\end{tabular}

\ Systematical error $\sim 9 \%$ with $300 \mathrm{pb}^{-1}$ 


\section{$B^{+} \rightarrow J / \psi K^{+}$Cross Section}

$\downarrow$ Reference channel

\ Di-muon $J / \psi$ trigger, $\epsilon^{\text {trig }}=82 \%$

$\downarrow J / \psi$ reconstruction: $\epsilon_{J / \psi}^{r e c}=55.8 \%$ $p_{T}^{\mu_{1}}>6 \mathrm{GeV}, p_{T}^{\mu_{2}}>3 \mathrm{GeV}$ displaced vertex $(>100 \mu \mathrm{m}$ in $\mathrm{xy})$

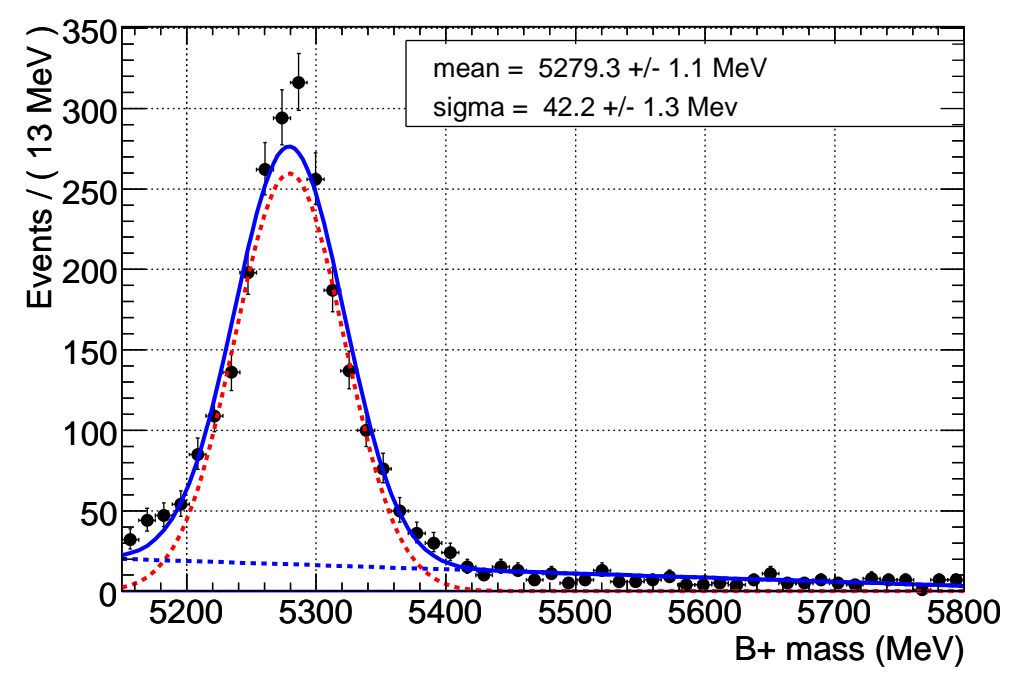

$\downarrow B^{+}$reconstruction: $J / \psi+1$ track $\left(p_{T}>1.5 \mathrm{GeV},\left|d_{0}\right| / \sigma_{d_{0}}>1\right)$ displaced vertex $\left(>100 \mu \mathrm{m}\right.$ in xy), mass in $\pm 120 \mathrm{GeV}$ around $m_{B^{+}}$

$\downarrow \epsilon^{t o t a l}=(29.8 \pm 0.84) \% \quad \sigma\left(m_{B^{+}}\right)=(42.2 \pm 1.3) \mathrm{MeV}$

^ with $13.2 \mathrm{pb}^{-1}(\sim 2100$ signal events $):$

cross section to $\sim 3 \% \quad$ mass resolution $\sim 3 \%$ signal lifetime to $\sim 2 \%$ 


\section{Heavy Quarkonia - Motivation}

- Narrow resonances useful for detector studies (trigger and detector calibration and alignment) and on-line monitoring

$\downarrow$ Background for many other processes at LHC

- Theoretical interest: production mechanism still not understood, testbed for QCD in perturbative and non-perturbative regimes
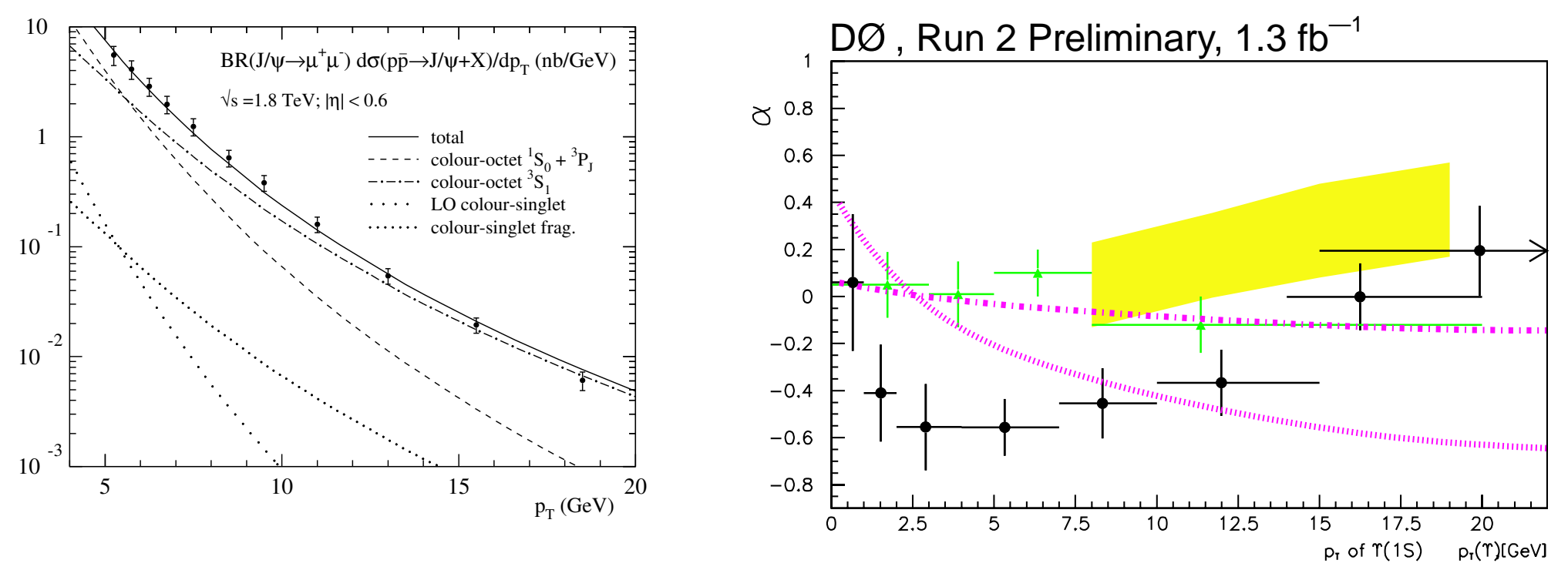


\section{Quarkonia Cross Sections}
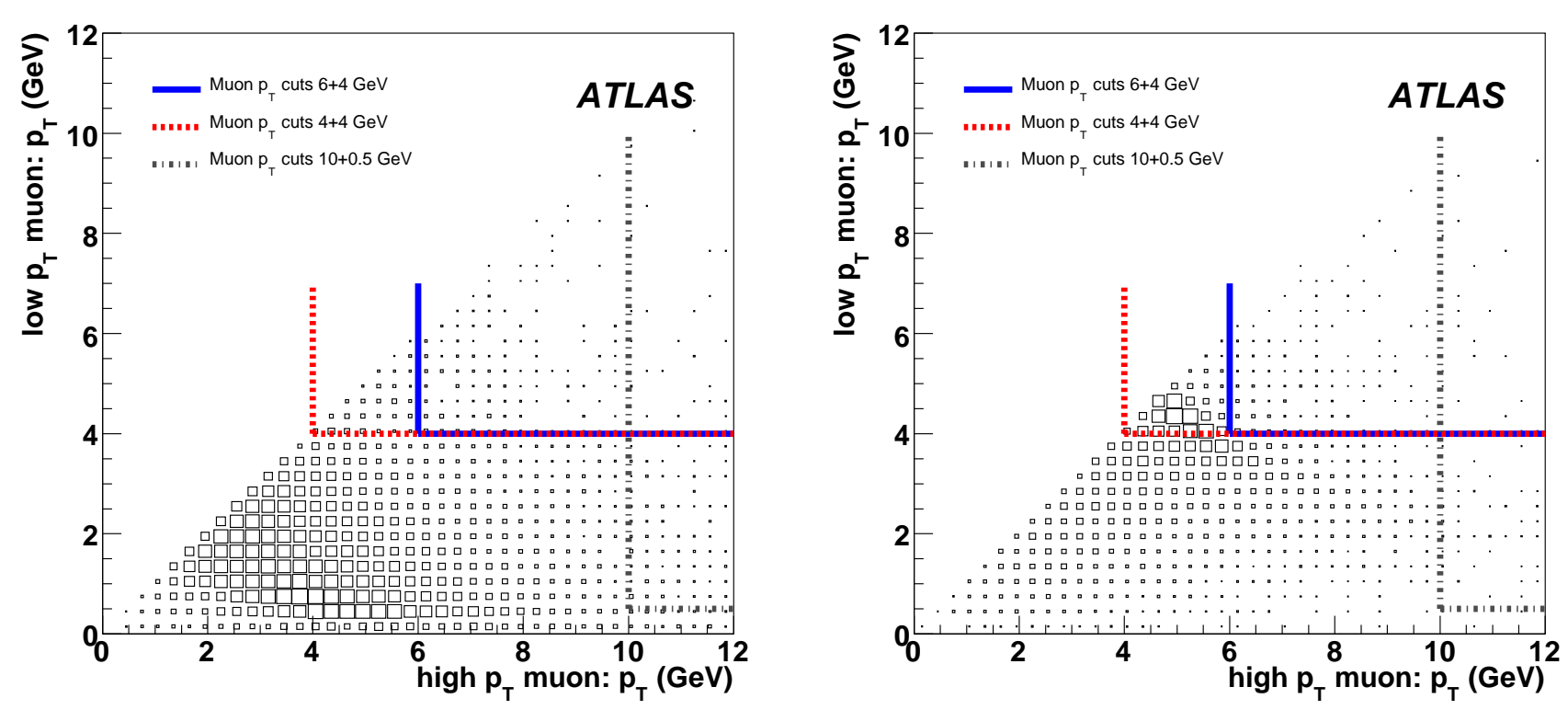

- Di-muon triggers: LVL1 2MU6 and 1MU4

\ Single-muon trigger with $p_{T}>10 \mathrm{GeV}$

\begin{tabular}{|l|c|c|c|}
\hline MC cuts & $6+4 \mathrm{GeV}$ & $4+4 \mathrm{GeV}$ & $10+0.5 \mathrm{GeV}$ \\
\hline$\sigma(J / \psi)$ & $23 \mathrm{nb}$ & $28 \mathrm{nb}$ & $23 \mathrm{nb}$ \\
$\sigma(\Upsilon)$ & $5.2 \mathrm{nb}$ & $48 \mathrm{nb}$ & $2.8 \mathrm{nb}$ \\
\hline
\end{tabular}




\section{Reconstruction of Prompt Quarkonia}

$\downarrow \mu$ tracks from primary vertex $\quad \mathcal{L}=10^{31} \mathrm{~cm}^{-2} \mathrm{~s}^{-1}$

$\checkmark$ Pseudo-proper time $<0.2 \mathrm{ps}$

$\downarrow$ Mass windows: $m_{J / \psi}^{P D G} \pm 300 \mathrm{MeV} \quad m_{\Upsilon}^{P D G} \pm 1 \mathrm{GeV}$
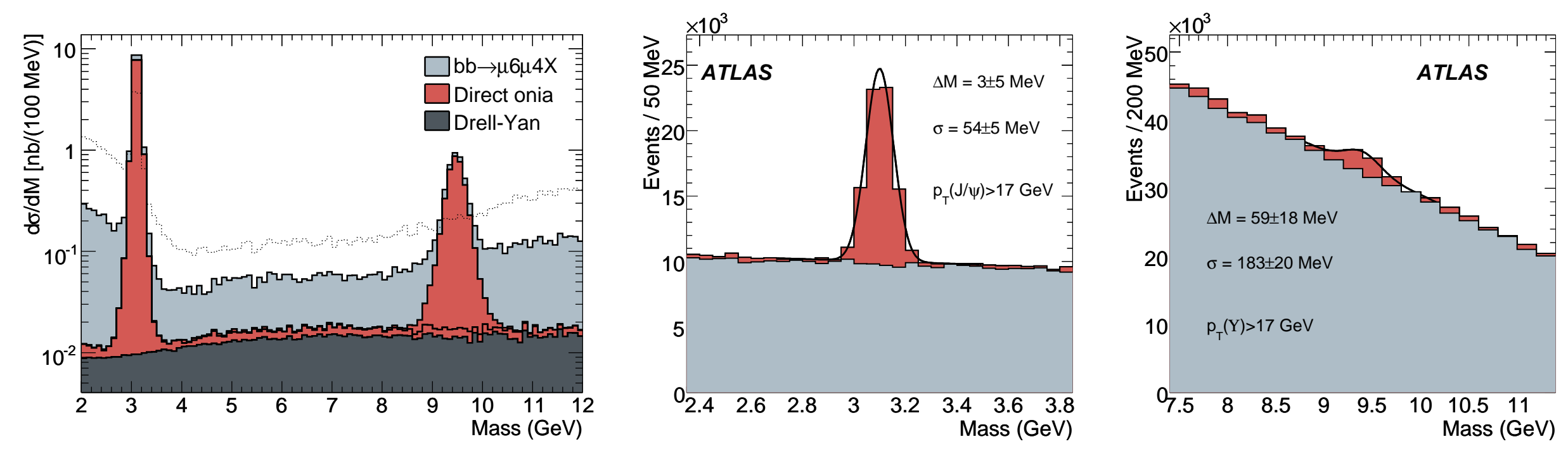

\begin{tabular}{|l|c|c|c|c|}
\hline with $10 p b^{-1}$ & \multicolumn{2}{|c|}{ Number of events } & \multicolumn{2}{c|}{ S/B } \\
\hline & $J / \psi$ & $\Upsilon$ & $J / \psi$ & $\Upsilon$ \\
\hline Di-muon trigger & 150000 & 25000 & 60 & 10 \\
Single-muon trigger & 160000 & 20000 & 1.2 & 0.05 \\
\hline
\end{tabular}




\section{Quarkonium Spin Alignment}

Can be determined by measuring the polarisation parameter $\alpha$ $\alpha=0$ unpolarised, $=+1$ transverse, $=-1$ longitudinal

$\downarrow$ Different models predict different $p_{T}$ dependencies

$\checkmark$ Different acceptances for di-muon and single-muon samples

$$
\frac{d N}{d \cos \theta^{*}}=C \frac{3}{2 \alpha+6}\left(1+\alpha \cos ^{2} \theta^{*}\right)
$$
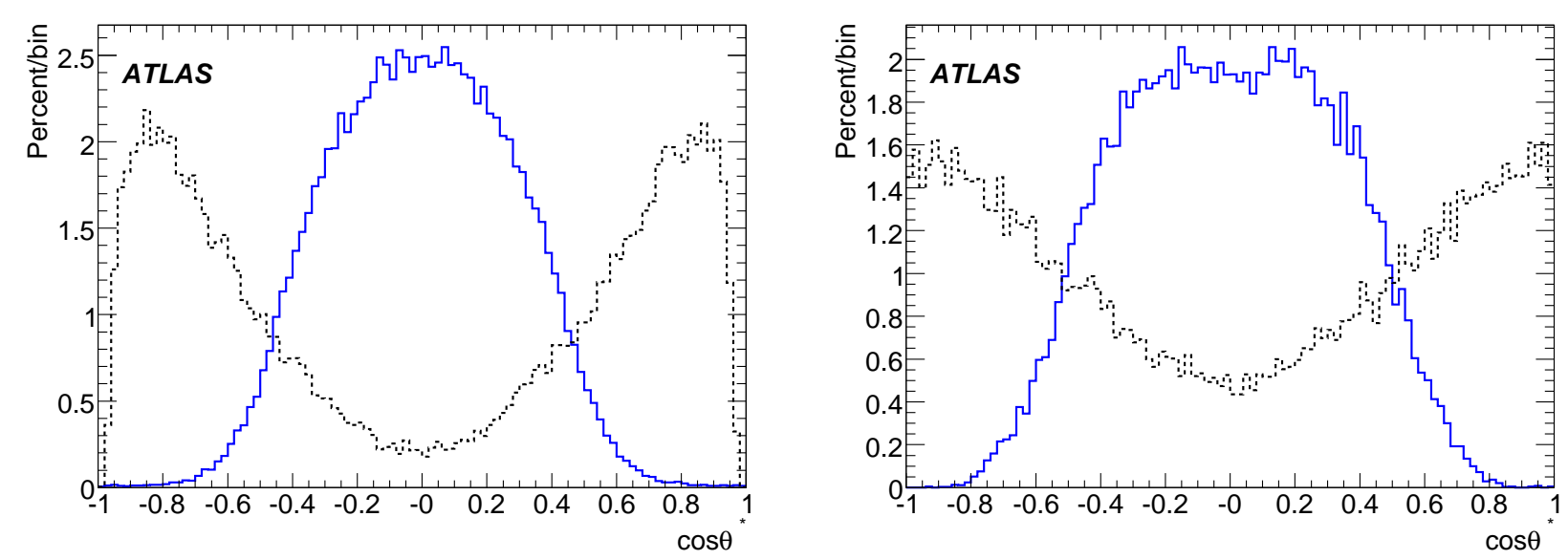


\section{Spin-alignment Measurement}

Unpolarised $J / \psi$ sample $\left(10 \mathrm{pb}^{-1}\right)$

$\checkmark$ Split into 6 bins of $p_{T}$

- Measure reconstructed distributions

$\checkmark$ Correct for acceptances and efficiencies
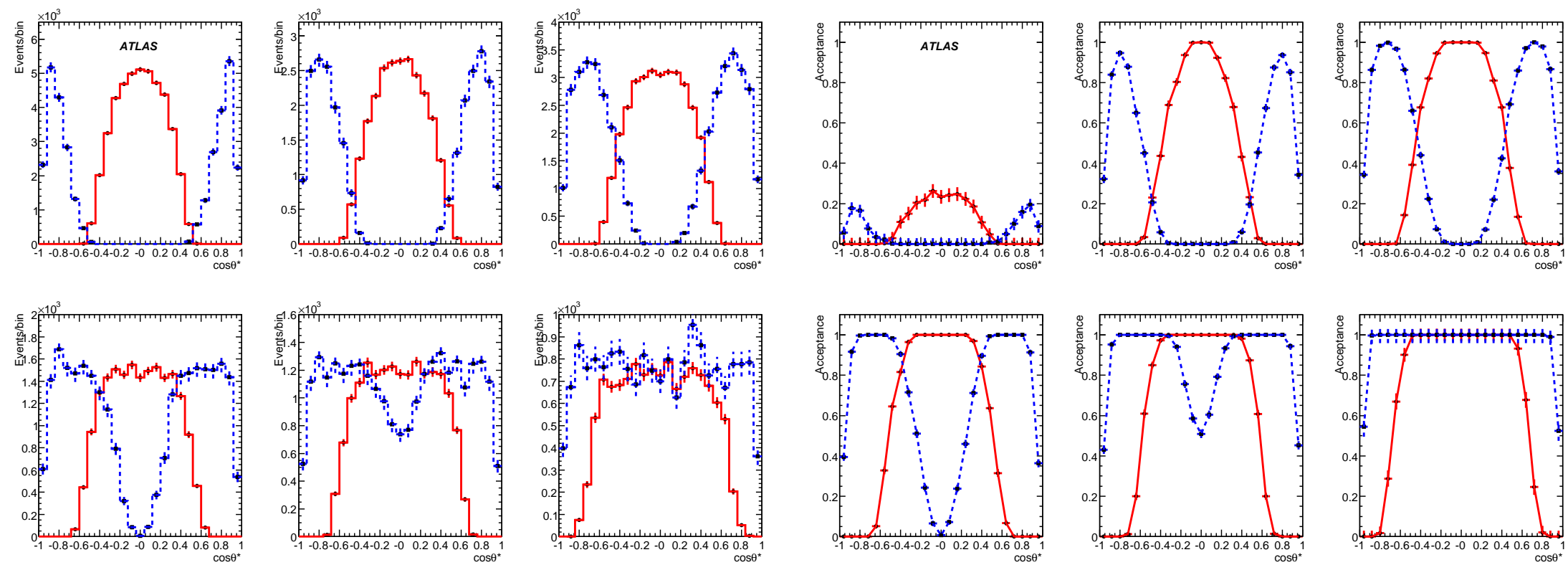

"Measured" distributions

Acceptance and efficiency

$p_{T}$ bins: $9-12 \mathrm{GeV}, 12-13 \mathrm{GeV}, 13-15 \mathrm{GeV}, 15-17 \mathrm{GeV}, 17-21 \mathrm{GeV},>21 \mathrm{GeV}$ 


\section{Spin-alignment Measurement}

Both samples normalised to each other using overlapping high $p_{T}$ events Use pre-defined acceptance mask to combine the two datasets

Fit to the corrected distributions
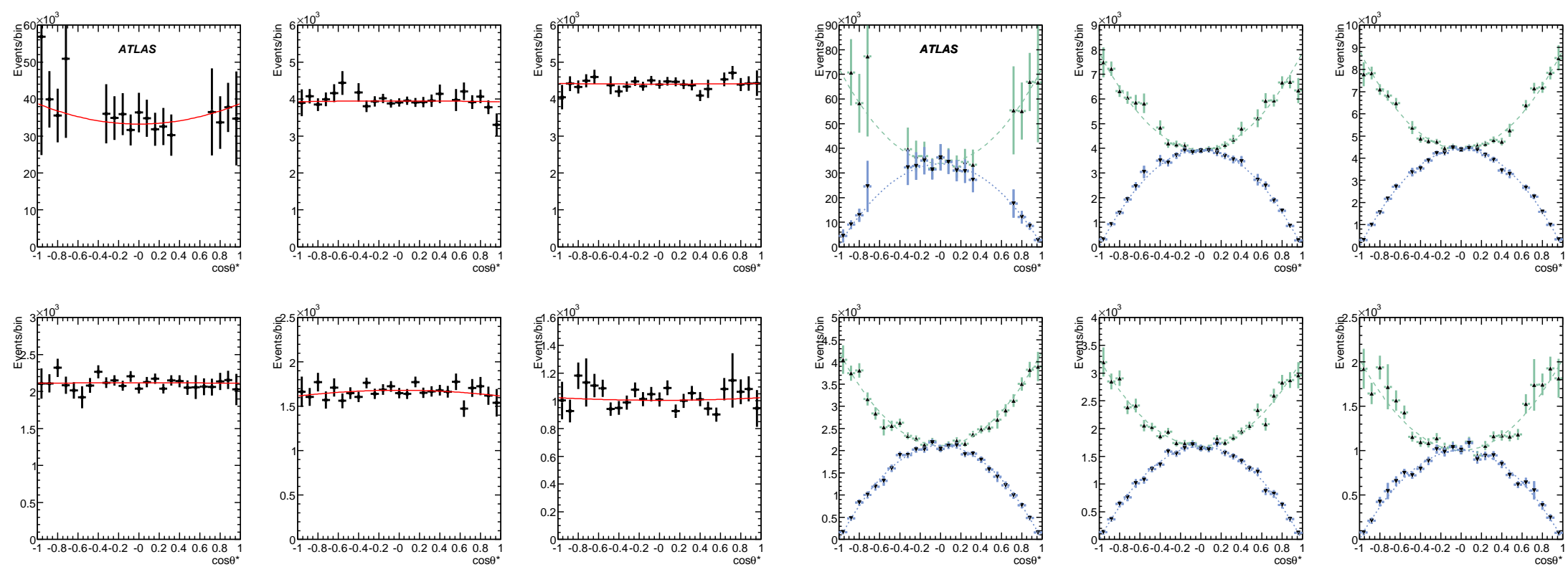

Precision in $\alpha$ with $10 \mathrm{pb}^{-1}: 0.02-0.06$ for $J / \psi$ and 0.2 for $\Upsilon$ 


\section{Decays of $\chi_{c}$ And $\chi_{b}$}

$\chi_{c} \rightarrow J / \psi\left(\mu^{+} \mu^{-}\right) \gamma$

४ combine $J / \psi$ with a soft $\gamma$

- $\cos \alpha(J / \psi, \gamma)>0.97$

- $m_{\mu \mu \gamma}-m_{\mu \mu}$ in $(200,700) \mathrm{MeV}$

- simultaneous fit to 3 Gaussians and a quadratic background

$\downarrow \epsilon^{r e c}=4 \%$, resolution $\sim 40 \mathrm{MeV}$

^ observable with $10 \mathrm{pb}^{-1}$

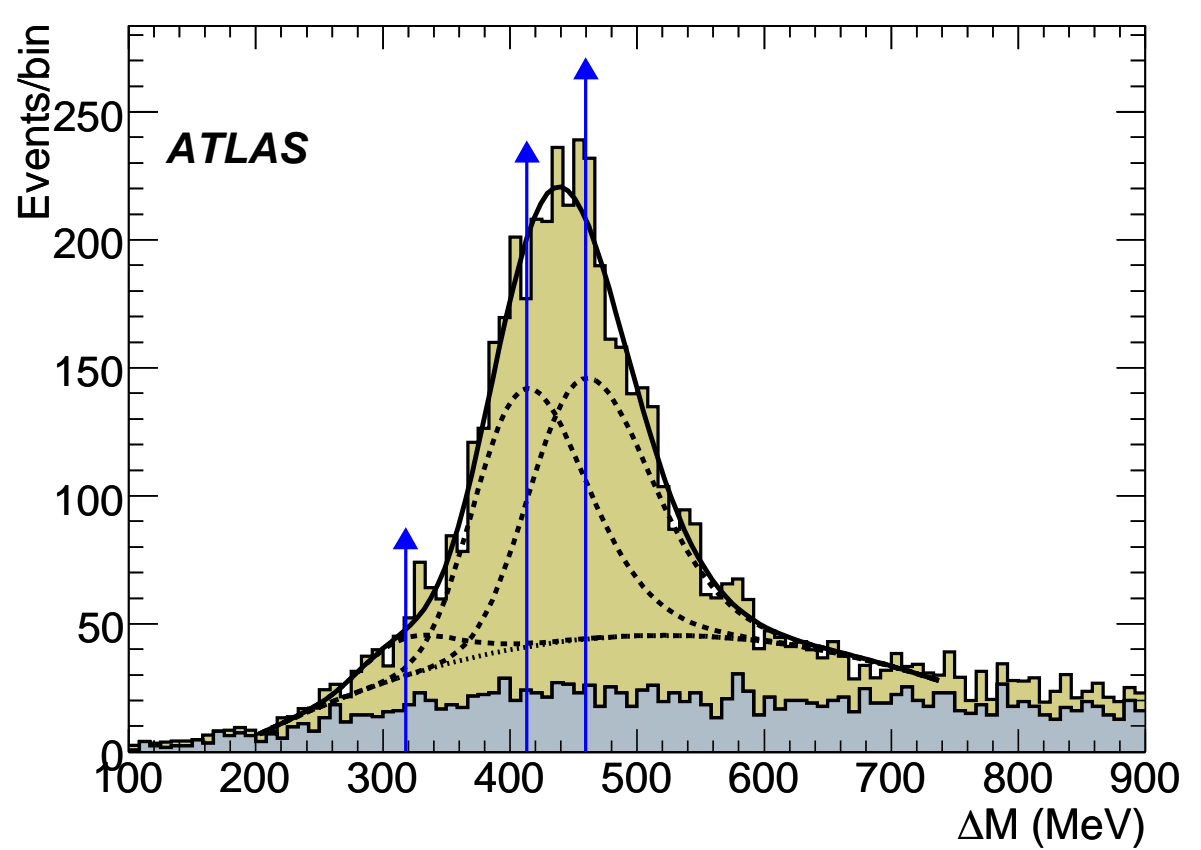

$\chi_{b} \rightarrow \Upsilon\left(\mu^{+} \mu^{-}\right) \gamma$

$\gamma$ much softer, $\epsilon^{r e c}=0.03 \% \rightarrow$ need $\sim 1 \mathrm{fb}^{-1}$ to observe

$\chi_{b} \rightarrow J / \psi\left(\mu^{+} \mu^{-}\right) J / \psi\left(\mu^{+} \mu^{-}\right)$

$\epsilon^{\text {total }} \sim 0.8 \% \rightarrow$ expect $\sim 100$ events with $10 \mathrm{fb}^{-1}$ 


$$
J / \psi \rightarrow e^{+} e^{-} \text {And } \Upsilon \rightarrow e^{+} e^{-} \text {Decays }
$$

$J / \psi$ and $\Upsilon$ one of the main sources of isolated electrons in early data

$\downarrow$ Ideal for studies of the trigger and offline performance

$\checkmark$ Calibration of the EM calorimeter

$\downarrow$ EM trigger: LVL1 2EM3 $\left(\mathcal{L}=10^{31} \mathrm{~cm}^{-2} \mathrm{~s}^{-1}\right) \quad \epsilon=27 \%$ ID tracks reconstructed in LVL1 Rol

b-trigger: $\operatorname{LVL1} 1 \mathrm{MU} 4\left(\mathcal{L}=10^{31}\right), 1 \mathrm{MU} 6\left(\mathcal{L}=10^{31}-10^{33}\right) \quad \epsilon=88 \%$ ID tracks reconstructed in whole ID $\left(\mathcal{L}=10^{31}\right)$, in LVL1 Rol $\left(\mathcal{L}=10^{33}\right)$

\section{Electron reconstruction:}

$\checkmark$ strict quality cuts on ID tracks

$\checkmark$ bremsstrahlung recovery

$\checkmark$ veto conversions

$\downarrow$ tracks extrapolated to EM calorimeter, $E / p>0.7$

Electron identification:

$\downarrow$ isolated electrons: based on shower shapes, $\epsilon \sim 65 \%$, pion rejection $\sim 900(740)$ for $J / \psi(\Upsilon)$

$\downarrow$ electrons from $b$ : core of the EM shower used, $\epsilon \sim 80 \%$, pion rejection $\sim 1300$ 


\section{$J / \psi \rightarrow e^{+} e^{-}$And $\Upsilon \rightarrow e^{+} e^{-}$Decays}

$J / \psi \rightarrow e^{+} e^{-}$and $\Upsilon \rightarrow e^{+} e^{-}$reconstruction:

- Momentum measurement from ID, energy from EM calorimeter

$\checkmark$ displaced vertex (> $250 \mu \mathrm{m}$ in $\mathrm{xy})$

\5\% reconstructed within $\pm 200 \mathrm{MeV}$ of $m_{J / \psi}$ (47\% w/o brem recovery)
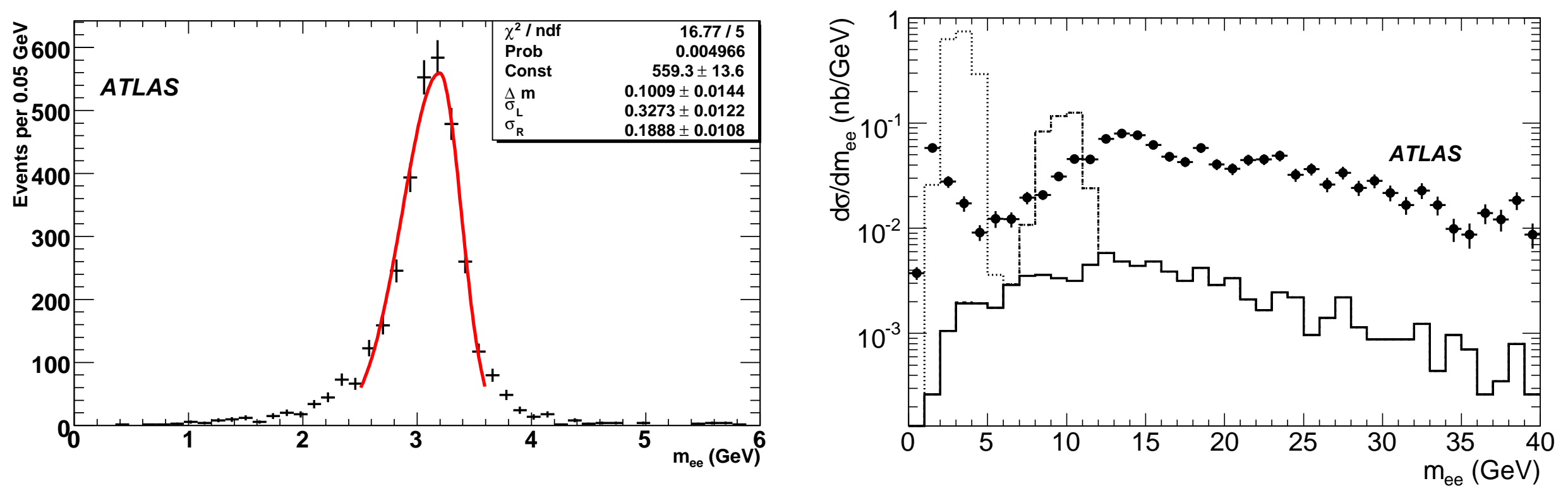

Expected events with $100 \mathrm{pb}^{-1}\left(\mathcal{L}=10^{31} \mathrm{~cm}^{-2} \mathrm{~s}^{-1}\right)$ :

$200000 \mathrm{~J} / \psi, 43000 \Upsilon$ (2EM3 trigger) and $19000 \mathrm{~J} / \psi$ (1MU6 trigger) 


\section{Conclusions}

ATLAS will measure beauty and onia production cross sections via $\mu X$ and $J / \psi X$ decays

$\checkmark \sigma, d \sigma / d p_{T}, d \sigma / d \eta, \sigma_{B^{+} \rightarrow J / \psi K^{+}}$

$\checkmark$ both $J / \psi \rightarrow \mu^{+} \mu^{-}$and $J / \psi \rightarrow e^{+} e^{-}$studied

$\checkmark$ early data should provide sufficient statistics

Perform $J / \psi$ and $\Upsilon$ polarisation studies

Many more tests of QCD expected

ATLAS has a rich B-physics programme (not covered here)

First data later this year! 


\section{ATLAS CSC Notes}

Beauty Production Cross Section Measurements with Early LHC Data: B-Physics Reference Channel $B^{+} \rightarrow J / \psi K^{+}$and Inclusive Methods

M. zur Nedden, C. Petridou, C. Anasopoulos, S. Jetter, O. M. Kind, M. Volkmann

Heavy quarkonium physics with early ATLAS data

T. Alexopoulos, F. Antoniou, E. Etzion, E. N. Gazis, J. Ginzburg, V. Kartvelishvili, D. Price

Reconstruction of $J / \psi \rightarrow e^{+} e^{-}$and $J / \psi \rightarrow e^{+} e^{-}$decays

T. Berger-Hryn'ova, E. Bouhova-Thacker, J. Cochran, F. Derue, A. Kaczmarska, V. Kartvelishvili, J. Kirk, E. Lytken, A. Nelson 